\title{
Specific length and structure rather than high thermodynamic stability enable regulatory mRNA stem- loops to pause translation
}

Chen Bao, Mingyi Zhu, Inna Nykonchuk, Hironao Wakabayashi, David H.

Mathews and Dmitri N. Ermolenko

Department of Biochemistry \& Biophysics at School of Medicine and Dentistry and Center for RNA Biology, University of Rochester, Rochester, NY, USA

To whom correspondence may be addressed: Dmitri N. Ermolenko (dmitri ermolenko@urmc.rochester.edu). 


\section{SUMMARY}

Translating ribosomes unwind mRNA secondary structures by three basepairs each elongation cycle. Despite the ribosome helicase, certain mRNA stem-loops stimulate programmed ribosomal frameshift by inhibiting translation elongation. Here, using mutagenesis, biochemical and single-molecule experiments, we examine whether high stability of three basepairs, which are unwound by the translating ribosome, is critical for inducing ribosome pauses. We find that encountering frameshift-inducing mRNA stem-loops from the $E$. coli dnaX mRNA and the gag-pol transcript of Human Immunodeficiency Virus (HIV) hinders A-site tRNA binding and slows down ribosome translocation by $15-20$ folds. By contrast, unwinding of first three basepairs adjacent to the mRNA entry channel slows down the translating ribosome by only $2-3$ folds. Rather than high thermodynamic stability, specific length and structure enable regulatory mRNA stem-loops to stall translation by forming inhibitory interactions with the ribosome. Our data provide the basis for rationalizing transcriptome-wide studies of translation and searching for novel regulatory mRNA stem-loops. 


\section{Introduction}

During translation elongation, the ribosome translocates along mRNA one codon at a time.

The narrow mRNA channel of the small ribosomal subunit ${ }^{1-3}$ accommodates mRNA only when

it is not basepaired. Hence, translation requires unwinding of mRNA secondary structure.

Indeed, the translating ribosome has been demonstrated to be an efficient helicase ${ }^{4,5}$ that unwinds three basepairs per translocation step. However, it remains unclear to what extent mRNA stem-loops reduce the rate of translation elongation relative to translation along unpaired mRNA. Because mRNAs have a propensity to form intramolecular baseparing interactions and were shown to fold into extensive secondary structures in live cells ${ }^{6-9}$, elucidating the effects of mRNA stem-loops on translation elongation has important implications for translation regulation.

Several published studies vary in their estimates for the magnitude of translation elongation slowdown induced by mRNA secondary structures ${ }^{5,10-13}$. For instance, optical tweezer experiments suggested that ribosome translocation through three consecutive GC basepairs is only 2-3-fold slower than translocation along unpaired RNA ${ }^{10,12}$. Consistent with these data, several transcriptome-wide ribosome profiling analyses performed in yeast and E. coli cells indicated that most of the secondary structure elements within coding regions of mRNA do not produce detectable ribosome pauses or significantly change translational efficiency ${ }^{14,15}$.

In contrast to aforementioned reports demonstrating modest impact of mRNA secondary structure on translation rate, a ribosome profiling study performed in E. coli revealed strong negative correlation between translational efficiency and the presence of extensive intramolecular basepairing interactions in ORFs suggesting that both initiation and elongation 
phases of protein synthesis can be regulated by mRNA secondary structure ${ }^{16}$. Another study suggested that the effects of mRNA secondary structure on translational efficiency measured by ribosome profiling may be masked by the presence of codons that are decoded by abundant tRNAs in the structured regions of ORFs ${ }^{17}$. These studies suggest that mRNA secondary structure may strongly affect the translation elongation rate.

In addition to adjusting the translation elongation rate, certain RNA stem-loop structures were found to induce ribosome stalling that results in accumulation of truncated polypeptides ${ }^{18}$ and No-Go mRNA decay ${ }^{19}$. Furthermore, certain mRNA stem-loops and pseudoknots are known to trigger programmed translational pauses $^{20}$ and stimulate -1 programmed ribosomal frameshifting (PRF) by slowing down the ribosome ${ }^{21}$. -1 PRF plays an important role in regulating synthesis of bacterial, viral and eukaryotic proteins, including translation of gag-pol polyprotein of Human Immunodeficiency Virus (HIV) ${ }^{22}$ and C-terminally extended polyprotein in SARS-CoV-2 coronavirus, which caused the COVID-19 pandemic ${ }^{23,24}$.

-1 PRF requires the presence of two signals in an mRNA: the heptanucleotide slippery sequence NNNWWWN (where $\mathrm{N}$ can be any nucleotide and $\mathrm{W}$ is either $\mathrm{A}$ or $\mathrm{U}$ ) and a downstream frameshift-inducing hairpin or a pseudoknot called the frameshift stimulating sequence $(\mathrm{FSS})^{25}$. The slippery sequence allows cognate pairing of the P-site and A-site tRNAs in both 0 and -1 frames and thus makes frameshift thermodynamically favorable ${ }^{26}$. A number of previous studies demonstrated that when the slippery sequence is bound in the $\mathrm{P}$ and A sites of the ribosome and the FSS is positioned at the entry to mRNA tunnel of the small subunit, the rate of tRNA/mRNA translocation decreases by approximately an order of magnitude ${ }^{11,27-31}$. We also recently demonstrated that, when positioned 11-12 nucleotides 
downstream of the P-site codon, FSSs can transiently escape the ribosome helicase and inhibit A-site tRNA binding by docking into the A site of the small (30S) ribosomal subunit ${ }^{31}$. Hence, the FSS slows down the ribosome through two alternative pathways: inhibiting translocation and A-site tRNA binding.

It is commonly assumed that mRNA stem-loops and pseudoknots induce -1 PRF and ribosome pausing due to their high overall thermodynamic stability ${ }^{21,32,33}$. However, the translating ribosome unwinds only three basepairs at a time and thus is unable to "sense" the overall thermodynamic stability of mRNA stem-loops or pseudoknots. Consistent with this idea, optical tweezer experiments demonstrated that when GC content remains constant throughout very long stem-loops, the ribosome can unwind dozens basepairs with a seemingly uniform rate $^{5}$. It has also been hypothesized that rather than the total stability of the FSS, high local thermodynamic stability of basepairs positioned at the entrance to the mRNA tunnel of the small subunit that are first unwound by the ribosome explain the FSS-induced ribosome pausing $^{32,34,35}$.

Here, we investigate to what degree high thermodynamic stability of the three basepairs, which are unwound by the translating ribosome, accounts for translation pausing mediated by frameshift-inducing mRNA stem-loops. We hypothesize that in addition to overall and local thermodynamic stability, other structural properties enable stem-loops to induce ribosome pausing. To test these hypotheses, we examine how sequence and structure perturbations of frameshift-inducing stem-loops affect their ability to slow down translation elongation.

We find that unwinding of three basepairs per elongation cycle only moderately affects the rate of translation elongation. By contrast, when encountered by the translating ribosome, 
frameshift-inducing stem-loops produce an order-of-magnitude longer translational pause.

Our results indicate that specific length and the presence of the loop are important for inducing long ribosome pauses. Hence, our findings offer new insights into translational control by mRNA secondary structures and provide rationale for the discovery of previously unknown regulatory secondary structure elements at transcriptome-wide level.

\section{Results}

We used a combination of smFRET and biochemical assays to investigate properties of frameshift-inducing stem-loops from E. coli $d n a X$ mRNA and the gag-po/ transcript of HIV that are important for inducing ribosome pausing and $-1 \mathrm{PRF}$. The dnaX mRNA encodes the $\mathrm{T}$ and Y subunits of DNA polymerase ${ } I^{36}$. The slippery sequence AAAAAAG and a downstream 11 basepair-long FSS mRNA hairpin, which are separated by a five nucleotide-long spacer, program -1 PRF that produces $\mathrm{Y}$ subunit with $50-80 \%$ efficiency $^{29,32,37}$ (Fig. 1). The 12 basepair-long RNA hairpin from HIV in combination with the slippery sequence UUUUUUA, induces -1 PRF with 5-10\% efficiency to yield the Gag-Pol polyprotein ${ }^{34,36}$. mRNAs containing the slippery sequence and HIV FSS undergo frameshifting in bacterial (E. coli) ribosomes in vitro and in vivo at frequencies comparable to those observed for HIV frameshifting in eukaryotic translation systems $\mathrm{s}^{38-41}$ providing evidence for a common mechanism of frameshifting and ribosomal pausing induced by FSS in bacteria and eukaryotes. Hence, the FSS from HIV can be studied in E. coli system.

To probe properties of FSS stem-loops that are essential for inhibiting translocation and Asite tRNA binding, we introduced mutations into the FSS in model mRNAs that were derived from the E. coli dnaX and HIV gag-pol transcripts (Fig. 1, Supplementary Table 1) ${ }^{31}$. Frameshift 
changes the spacing between the P-site codon and the FSS and may also partially alleviate ribosome pausing. To delineate the effect of the FSS RNA hairpin on ribosome pausing from frameshifting, slippery codons in sequences derived from HIV and dnaX mRNAs were replaced with "non-slippery" codons as previously described ${ }^{31,42,43}$ (Fig. 1a). Mutations in the slippery sequence have been shown to decrease frameshifting efficiency to low or undetectable levels ${ }^{26,29,32,37,44}$. Each model mRNA contained the Shine-Dalgarno (SD, ribosome-binding site) sequence, a short open reading frame (ORF) and a downstream FSS. In addition, upstream of the SD sequence, each mRNA contained a 25 nucleotide-long sequence complementary to a biotin-conjugated DNA oligonucleotide that is used to tether the mRNA to a microscope slide for smFRET experiments (Figure 1a, Supplementary Table 1).

The stability of three basepairs of the FSS first unwound by the ribosome has no effect on A-site tRNA binding.

We examined the FSS-mediated inhibition of the A site tRNA binding using a filter-binding assay as previously described ${ }^{31}$. Using this approach, we found that when positioned 11 or 12 nucleotides (but not 13 nucleotides) downstream of P-site codon, the FSS from HIV and dnaX strongly hinders binding of aminoacyl(aa)-tRNA to the A site ${ }^{31}$. Here, we measured kinetics of tRNA binding to the A site of ribosomes, which contained P-site N-Ac-PhetRNA $^{\text {Phe }}$ and were programmed with dnaX or HIV mRNA variants. In these ribosomal complexes, the FSS was positioned 11 nucleotides downstream from the P-site (UUC) codon (Fig. 1a). Throughout the manuscript, the position of the FSS is described relative to the first nucleotide of the P-site codon. For example, positioning 11 nucleotides downstream 
of the P-site codon means that the first nucleotide of the P-site codon and the first nucleotide of the FSS are +1 and +12 , respectively (Fig. 1a).

The dnaX- or HIV-ribosome complexes were incubated with $\left[{ }^{3} \mathrm{H}\right] \mathrm{Tyr}-\mathrm{tRNA}{ }^{\mathrm{Tyr}}$ and EFTu•GTP (Fig. 2a). Next, the ribosomes were loaded onto a nitrocellulose filter and unbound $\left[{ }^{3} \mathrm{H}\right]$ Tyr-tRNA ${ }^{\text {Tyr }}$ was washed away. Consistent with our previously published data ${ }^{31}$, both wild-type dnaX and wild-type HIV FSSs severely slowed down the rate of $\left[{ }^{3} \mathrm{H}\right] \mathrm{Tyr}$-tRNA ${ }^{\text {Tyr }}$ binding. The apparent pseudo first order rates of tRNA binding were 0.2 (HIV, Figure 3a) and 0.3 (dnaX, Fig. 3b) $\mathrm{min}^{-1}$, respectively. When ribosomes were programmed with truncated mRNAs lacking FSSs (either dnaX $\Delta \mathrm{FSS}$ or HIV $\Delta \mathrm{FSS}$ ), the rate of $\left[{ }^{3} \mathrm{H}\right] \mathrm{Tyr}$-tRNA ${ }^{\mathrm{Tyr}}$ binding was too fast to be measured by the filter-binding assay requiring manual mixing of ribosome and tRNA.

Because the ribosome unwinds three pairs at a time, we investigated how the stability of the three bottom basepairs of the FSS affect tRNA binding. Two (in HIV) or three (in dnaX) G-C pairs at the bottom of respective FSSs were replaced with less stable G-U or A-U pairs to create HIV destabilized I and dnaX destabilized mRNAs (Fig. 1b). These mutations were predicted by the RNAstructure software ${ }^{45}$ to decrease the stability of the bottom three basepairs in HIV and dnaX FSSs by 4.7 and $6.2 \mathrm{kcal} / \mathrm{mol}$, respectively. However, probabilities of forming each basepair in HIV and dnaX stem-loops were predicted to be over $80 \%$ because of high overall stability of both HIV and dnaX mutant FSSs (-19.0 and -12.9 $\mathrm{kcal} / \mathrm{mol}$, respectively) and because sequences were designed to avoid pairing with the rest of the mRNA (Supplementary Fig. 1). To further destabilize the bottom of the HIV FSS, an additional two G-C basepairs were replaced with less stable A-U pairs to create HIV 
destabilized II mRNA (Fig. 1b, Supplementary Fig. 1). Relative to the wild-type FSS, the stability of the first five FSS basepairs in HIV destabilized II mRNA decreased by 8.6 $\mathrm{kcal} / \mathrm{mol}$ while overall stability of the FSS remained high $(-15.0 \mathrm{kcal} / \mathrm{mol})$.

A-site tRNA binding was equally slow $\left(0.2 \mathrm{~min}^{-1}\right)$ in the presence of the wild-type, destabilized I and destabilized II HIV FSSs (Fig. 3a). Likewise, experiments with dnaX mRNAs demonstrated that the destabilization of the bottom three basepairs has no effect on A-site tRNA binding (Fig. 3b). These results indicate that the stability of basepairs of the FSS that are first unwound by the ribosome does not affect the ability of HIV and dnaX FSS to inhibit A-site tRNA binding.

\section{The stem length and the presence of the loop are important for the ability of the}

\section{FSS to inhibit A-site tRNA binding.}

To explore the role of stem length, we truncated the wild-type HIV FSS by deleting the upper six basepairs adjacent to the loop while leaving bottom six basepairs intact (Fig. 1b). Importantly, the fractions of mRNA molecules forming all basepairs in the truncated stemloop was predicted to be over $99 \%$ providing evidence that truncated HIV FSS variant is formed despite the decrease in overall thermodynamic stability from -23.6 kcal/mol (wild-type HIV) to $-11.6 \mathrm{kcal} / \mathrm{mol}$. In the HIV mRNA with truncated FSS, the local stability of bottom basepairs first unwound by the ribosome was equal to the stability of these basepairs in the wild-type FSS. When ribosomes were programmed by the HIV truncated FSS mRNA instead of the HIV wild-type FSS mRNA, the pseudo first order rate constant for tRNA binding increased from 0.2 to $0.9 \mathrm{~min}^{-1}$ (Fig. 3a). This experiment indicates that in contrast to the 
stability of bottom basepairs, stem-loop length is an important property that appreciably affects the ability of stem-loops to inhibit tRNA binding.

To examine the contribution of the stem-loop topology to FSS-induced inhibition of tRNA binding, we truncated the HIV FSS upstream of the loop and annealed a 12-mer RNA oligo to create a "loopless" RNA bimolecular helix, which is identical to the stem of the HIV stem-loop. Likewise, by annealing an RNA oligo to the dnaX mRNA, which was truncated upstream of the FSS loop, the dnaX bimolecular helix similar to the stem of wild-type dnaX FSS was created (Fig. 1c). The dnaX bimolecular helix is predicted to have two structures in equilibrium with folding free energy changes of is $-20.3 \mathrm{kcal} / \mathrm{mol}$ and $-20.2 \mathrm{kcal} / \mathrm{mol}$ (Fig 1c, Supplementary Fig. 1). The conformational difference is whether the $C$ bulges in the mRNA (Fig 1c, Supplementary Fig. 1) or the trans oligonucleotide (more similar to the dnaX hairpin structure; Fig. 1c, Supplementary Fig. 1). The base of the helix has similarly high probability as the dnaX wild-type hairpin stem-loop. When compared to the stem-loops, the bimolecular helices lack the loops.

Based on the free energy change for the formation of the bimolecular helices, we calculated the equilibrium constants and concentrations of the annealed bimolecular helices (Supplementary Table 2). These assessments predict that at concentrations of mRNAs and RNA oligos used during the ribosome complex assembly, nearly $100 \%$ of mRNA molecules form bimolecular helixes. Furthermore, estimated dissociation rates ${ }^{46}$ (Supplementary Table 2) predict that both HIV and dnaX bimolecular helices remain stable within the duration of our biochemical and single-molecule experiments.

When ribosomes were programed with either HIV biomolecular helix or dnaX biomolecular 
helix mRNA, no inhibition of A-site tRNA binding was observed, i.e. the rate of tRNA binding was too fast to be measured by filter-binding assay, which involves manual mixing of the ribosome and tRNA (Fig. 3a-b). Hence, our results show that the presence of a loop in the FSS may be essential for the inhibition of A-site tRNA binding.

Loop regions of the wild-type HIV (ACCA) and dnaX (UACCC) FSSs differ in both sequence and length. Hence, despite the importance of the presence of the loop for the FSS-induced inhibition of A-site tRNA binding, specific loop sequence and length are unlikely to play key roles in the FSS-mediated regulation of translation elongation. To further test this hypothesis, we replaced the original, 4 nucleotide-long ACCA loop of HIV FSS with an 8 nucleotide-long ACAGCAGA loop, which was predicted to not lower the probability of the base pairs in the stem-loop (Supplementary Fig. 1). This loop extension resulted in a two-fold increase in the rate of A-site tRNA binding when compared to the wild-type HIV FSS (Fig. 3a). The relatively modest effect of the loop extension on tRNA binding rate supports the idea that loop sequence and length are not critical for the ability of the FSS to inhibit A-site tRNA binding.

Taken together, our filter-binding data indicate that the specific stem length and the presence of the loop are important for the ability of the frameshift-inducing stem-loops to inhibit A-site tRNA binding while overall and local thermodynamic stability have negligible effects.

The overall and local thermodynamic stabilities of the FSSs moderately affect ribosomal translocation.

When positioned 11 or 12 nucleotides dowstream of the P-site codon, HIV and dnaX FSSs strongly inhibit A-site binding but do not completely block it as the A site eventually becomes filled during extended incubation of the ribsome with aminoacyl-tRNA and EF-Tu•GTP (Figure 
3). The resulting pre-translocation ribosomes can be employed to examine the effect of the FSSs on the translocation step of the elongation cycle.

We next used single-molecule miscroscopy (Fig. 2b) and all aformenetioned mRNA constructs (Fig. 1) to investigate the properties of HIV and dnaX FSSs, which are important for the inhibition of ribosomal translocation. Following aminoacyl-tRNA binding to the ribosomal A site and peptide-bond formation, the pre-translocation ribosome predominantly adopts the rotated $(\mathrm{R})$ conformation ${ }^{47-49}$. In this conformation, the small ribosomal subunit (the $30 S$ subunit in bacteria) is rotated by $\sim 9^{\circ}$ relative to the large subunit (the $50 \mathrm{~S}$ subunit) ${ }^{50-52}$, and two tRNAs adopt the intermediate hybrid states of binding ${ }^{53-55}$. EF-G-catalyzed mRNA/tRNA translocation on the small subunit is coupled to the reverse rotation of the ribosomal subunits, restoring the nonrotated (NR) conformation in the post-translocation ribosome ${ }^{47,49,56}$. Hence, ribosome translocation is accompanied by $\mathrm{R}$ to NR transition. To follow $R$ to NR transition, which is coupled to translocation, we measured single-molecule Förster resonance energy transfer (smFRET) between fluorophores attached to the 50 S protein bL9 and the $30 \mathrm{~S}$ protein $\mathrm{bS6}^{31}$ (Fig. 2b). The NR and R conformations of the ribosome have been shown to correspond to the 0.6 and 0.4 FRET states of S6-cy5/L9-cy3 FRET pair ${ }^{47,48}$.

The pretranslocation S6-cy5/L9-cy3 labeled ribosomes containing A-site peptidyl-tRNA $\left(N-A c-P h e-T y r-t R N A^{T y r}\right)$ and P-site deacylated tRNA ${ }^{\text {Phe }}$ were programed with a FSScontaining mRNA (Fig. 2b). In these complexes, the FSS was positioned $11 \mathrm{nt}$ downstream of the P-site codon. When the first nucleotide of the P-site codon is defined as +1 , the +11 nucleotide is the last mRNA residue bound in the mRNA tunnel of the small subunit ${ }^{1-3,57}$. The first nucleotide of the FSS $(+12)$ is positioned at the tunnel entrance (Fig. 1a). 
Consistent with previous reports ${ }^{48,58}$, when imaged in the absence of EF-G, pretranslocation ribosomes containing deacylated tRNA ${ }^{P h e}$ in the $\mathrm{P}$ site spontaniously fluctuate between R and NR conformations at rates of $0.8 \mathrm{~s}^{-1}$ (NR to R, Supplementary Fig. 2a) and 0.5 $\mathrm{s}^{-1}$ ( $\mathrm{R}$ to NR, Supplementary Fig. $2 \mathrm{~b}$ ). The $\mathrm{R}$ confromation is predominant as $95 \%$ of pretranslocation ribosomes spend less than $4 \mathrm{~s}$ in the NR confromation (Supplementary Fig. 2a). In contrast to these unproductive fluctuations, EF-G-induced translocation fixes the ribosome in the NR state ${ }^{48}$. To distinguish translocation from spontaneous unproductive fluctuations between R and NR conformations, we define EF-G-induced translocation as a transition from $\mathrm{R}$ to the stable NR state (i.e. 0.6 FRET state lasting over $4 \mathrm{~s}$ ).

In translocation measurements, at $10 \mathrm{~s}$ of imaging, EF-G.GTP was injected into the microscope slide bound with pretranslocation ribosomes (Fig. 2b, Fig. 4). The dwell time $T_{\text {trl(translocation) }}$ between the injection and the transition from R (0.4 FRET) to the stable NR $(0.6$ FRET) ribosomal conformations reflects the translocation rate (Fig. 4$)^{31}$.

When the HIV FSS was positioned 11 nucleotides downstream of the $\mathrm{P}$ site codon, translocation was so slow that most ribosomes remained in the $\mathrm{R}$ state until photobleaching of the acceptor dye. Under our experimental conditions, the rate of photobleaching was 0.03 $\mathrm{s}^{-1}$ (Supplementary Fig. 2c). To extend the lifetime of the acceptor fluorophore and detect translocation in most ribosomes, the excitation laser was switched off after the EF-G injection and switched back on either 40 or $60 \mathrm{~s}$ later (Fig. 4a). Varying the interval for which the excitation laser was switched off between 40 and $60 \mathrm{~s}$ did not significantly affect the distribution of $\mathrm{T}_{\mathrm{trl}}$ in ribosome population indicating that the number of ribosomes translocating during the laser shutoff was negligible (Supplementary Fig. 3a-b). $\mathrm{T}_{\mathrm{trl}}$ measured in ribosomes 
programmed with the HIV FSS was remarkably long (Fig. 4a) with median value of $73.3 \mathrm{~s}$ (Fig. 4b, Fig. 5a, Supplementary Fig. 3a-b). By contrast, when ribosomes were programmed with HIV mRNA lacking the FSS (HIV $\triangle$ FSS mRNA), median $T_{\text {trl }}$ was $2.8 \mathrm{~s}$ indicating that HIV FSS reduces the translocation rate by 25 -fold (Fig. 4c-d, Fig. 5a).

Next, we measured translocation kinetics in ribosomes programmed with dnaX mRNA, in which the dnaX FSS was positioned 11 nt downstream of the P-site (UUC) codon (Fig. 1a). Because of slow rate of translocation in the presence of the dnaX FSS, the excitation laser was switched off after the EF-G injection and switched back on either 40 or $60 \mathrm{~s}$ later to extend lifetime of the acceptor fluorophore. Similar to experiments with HIV mRNA, varying the laser shutoff time did not significantly affect the distribution of $T_{\text {trl }}$ in ribosome population indicating that the number of ribosomes translocating during the laser shutoff was negligible (Supplementary Fig. 3e-f). When compared to the mRNA lacking the FSS, dnaX FSS reduced the translocation rate 15 times as $\mathrm{T}_{\mathrm{trl}}$ changed from 5.1 (dnaX $\triangle \mathrm{FSS} \mathrm{mRNA}$, Fig. $5 \mathrm{~b}$, Supplementary Fig. 4d) to 73.4 s (dnaX wild-type FSS mRNA, Fig. 5b, Supplementary Fig. 4a).

To examine how the kinetics of translocation depend on the spacing between the P-site codon and the FSS, we made two HIV mRNA variants where the spacer between the P-site (UUC) codon was extended from 11 to 12 or 13 nucleotides (Fig. 1a, Fig. 4). Extending the Psite-FSS spacer from 11 to 12 nucleotides decreased median Ttrl from 73.3 to $28.6 \mathrm{~s}$ (Fig. $4 \mathrm{e}-$ f) while lengthening the the P-site-FSS spacer to 13 nucleotides further decreased median $T_{\text {trl }}$ to $16.9 \mathrm{~s}$ (Fig. 4g-h). Hence, extending the P-site-FSS spacer progressively accelerates translocations. Noteworthy, in the experiments with 12 and 13-nucleotide long P-site-FSS 
spacers, the excitation laser was not switched off at any point (Fig. 4e, g) because translocation rates were much faster than the acceptor photobleaching rate.

Assuming that all FSS nucleotides residing outside of the mRNA tunnel are basepaired, extending the P-site-FSS spacer from 11 to 12 or 13 nucleotides is expected to reduce the number of basepairs unwound upon a round of ribosome translocation from 3 to 2 or 1 , respectively. Hence, our data may indicate that the number of basepairs unwound by the ribosome determines the extent of translocation pausing induced by the FSS. Alternatively, the short (11 nucleotide) spacer restricts the FSS dynamics and thus entropically stabilizes FSS-ribosome intectaions that inhibit translocation.

To investigate how the stability of three lowest basepairs of the FSS that are first unwound by the ribosome affects FSS-mediated inhibition of translocation, we used mutant variants of HIV and dnaX mRNAs (Fig. 1b). These mRNA variants were also employed in filter-binding experiments described above (Fig. 3). Destabilization of the three (HIV FSS) or four (dnaX FSS) bottom basepairs of the FSS that are first unwound by the ribosome slightly accelerated translocation as $\mathrm{T}_{\mathrm{trl}}$ decreased from $73 \mathrm{~s}$ (both wild-type HIV and wild-type dnaX FSSs) to 41.1 s (HIV destabilized FSS I, Fig. 5a, Supplementary Fig. 5a) and 52.3 s (dnaX destabilized FSS, Fig. 5b, Supplementary Fig. 3 g,h, Supplementary Fig. 4b), respectively. Reducing the stability of the first five FSS basepairs in HIV destabilized II mRNA led to two-fold acceleration of translocation rate as median $\mathrm{T}_{\text {trl }}$ decreased from $73.3 \mathrm{~s}$ (wild-type HIV FSS) to $32.1 \mathrm{~s}$ (HIV destabilized FSS II) (Fig. 5a, Supplementary Fig. 5b).

When compared to the wild-type FSS HIV mRNA, distributions of $\mathrm{T}_{\mathrm{tr}}$ times observed in the presence of destabilized FSS I and II HIV mRNAs were markedly more heterogeneous 
(Supplementary Fig. 5a-b). These data likely indicate that destabilizing mutations introduced into HIV FSS and interactions of mutant FSS variants with the ribosome lead to structural heterogeneity of the stem-loop such as possible fraying of the stem-loop bottom basepairs. Nevertheless, the predominant peaks in $T_{\text {trl }}$ distributions observed with destabilized HIV FSS I and II mRNAs likely correspond to the slowest ribosomes bound with the fully intact FSS (Supplementary Fig. 5a-b).

Taken together, experiments with dnaX and HIV mRNA variants with destabilized bottom basepairs provide evidence that the stability of basepairs, which are first unwound by the translating ribosome, moderately contributes to the inhibition of translocation by the dnaX and HIV FSSs.

The stem length and the presence of the loop are important for the inhibition of translocation by the FSSs.

We next explored how stem length and the presence of the loop affect FSS-induced inhibition of ribosome translocation. In contrast to relatively weak effects of destabilization of bottom basepairs, truncation of the HIV FSS by deleting the upper six basepairs adjacent to the loop while leaving "bottom" six basepairs intact (Fig. 1), accelerated translocation rate by 7-fold as $\mathrm{T}_{\text {trl }}$ decreased from 73 to $11 \mathrm{~s}$ (Fig. 5a, Supplementary Fig. 5c). Importantly, in HIV mRNA with a truncated FSS the local stability of bottom basepairs first unwound by the ribosome was equal to the stability of these basepairs in the wild-type FSS. Furthermore, the overall thermodynamic stability of the truncated HIV FSS $(-11.6 \mathrm{kcal} / \mathrm{mol})$ was only slightly lower that the stability of HIV destabilized FSS II (-15 kcal/mol) and dnaX destabilized FSS ($12.9 \mathrm{kcal} / \mathrm{mol}$ ) (Figure 1b). Hence, the optimal stem length contributes more to the FSS ability 
to inhibit translocation than overall and local thermodynamic stabilities of the stem-loop.

Swapping the 4 nucleotide-long ACCA loop of HIV FSS with an 8 nucleotide-long ACAGCAGA loop did not change median Ttrl (Fig. 5a, Supplementary Fig. 3c-d, Supplementary Fig. 5d) indicating that altering length and sequence of the HIV FSS loop does not affect FSSinduced inhibition of translocation. By contrast, replacing the wild-type HIV and dnaX FSSs with "loopless" bimolecular helices that are identical to stems of HIV and dnaX FSSs (Fig. 1c) similarly accelerated the rate of translocation by $\sim 7$ fold (Fig. 5a-b). Median $T_{\text {trl }}$ decreased from $73 \mathrm{~s}$ (both wild-type HIV and dnaX FSSs) to $10.3 \mathrm{~s}$ (HIV bimolecular helix, Fig. 5a, Supplementary Fig. 5e) and 12.7 s (dnaX bimolecular helix, Fig. 5b, Supplementary Fig. 4c). These results show that the presence of the loop plays an important role in the FSS-induced inhibition of translocation.

Next, we tested whether another bimolecular RNA duplex that is not derived from a frameshift-inducing stem-loop has a similar effect on translocation as biomolecular helices derived from HIV and dnaX FSSs. To that end, a 16 nucleotide-long RNA oligo was annealed to an unstructured region of a model mRNA derived from the phage T4 gene 32 mRNA named $\mathrm{m} 291^{59}$ (Fig. 1a). Importantly, the overall thermodynamic stability of the m291 bimolecular helix (-24.5 $\mathrm{kcal} / \mathrm{mol})$ was comparable to the stabilities of biomolecular helixes derived from HIV and dnaX FSSs and was also slightly higher than the stabilities of wild-type HIV and dnaX FSSs (Figure 1c).

Similar to experiments with HIV and dnaX bimolecular helices, in pretranslocation ribosomes programed with m291 mRNA containing A-site peptidyl-tRNA (N-Ac-Met-PhetRNA $^{\text {Phe }}$ ) and P-site deacylated tRNA ${ }^{\text {Met }}$, the RNA oligo was positioned 11 nucleotides 
downstream of the P-site (AUG) codon (Figure 1a). When compared to translocation observed in m291 mRNA-programmed ribosomes in the absence of the RNA oligo (Fig. 6a), the presence of bimolecular helix reduced the rate of translocation by 2 fold (Fig. 6b). Although HIV, dnaX and m291 biomolecular helixes differed in sequence and stabilities of the three basepairs, which are adjacent to $30 \mathrm{~S}$ mRNA tunnel and unwound by the ribosome upon translocation, median values of dwell time $T_{\text {trl }}$ measured in the presence of these constructs were similar.

Remarkably, when compared to the mRNAs lacking the FSS (m291, HIV $\Delta$ FSS and dnaX $\triangle \mathrm{FSS}$ mRNAs), both truncated FSS and "loopless" bimolecular helix constructs reduced translocation rate $\sim 2-3$ fold. These results are consistent with previous optical tweezers experiments demonstrating that translocation through three GC basepairs is only 2-3-fold slower than translocation along a single-stranded codon ${ }^{10,12,13}$.

Taken together, our data show that unwinding of three basepairs during one round of translocation only moderately slows down the ribosome as evident from experiments with bimolecular helices. Hence, much stronger inhbition of translocation observed in the presence of wild-type HIV and dnaX FSSs are due to their specific structural properies rather than their high thermodynamic stabilities.

\section{Discussion}

We find that the total thermodynamic stablity of the FSSs and the local stablity of the basepairs adjacent to the entry of mRNA tunnel moderately influence ribosome pausing. Hence, instead of merely acting as a physical barrier for the translating ribosome, the FSS likely hinder protein synthesis by forming specific interactions with the ribosome. Consistent 
with this idea, the lack of correlation between total thermodynamic stability of the FSS and the efficiency of frameshifting has been observed in several studies ${ }^{60,61}$. In addition, while modest stabilization of the bottom basepairs of the HIV FSS has been shown to increase frameshifting efficiency, significant destabilization of the first three basepairs of the HIV FSS has been shown to have no effect on the efficiency of frameshifting ${ }^{34}$.

By contrast, destabilizing substitutions at the bottom of dnaX FSS have been shown by Atkins and colleagues to significantly decrease frameshifting efficiency measured by the $\beta$ galactosidase reporter ${ }^{32}$. The apparent discrepancy between that study and our data may be due to differences in model mRNA lengths. In contrast to short model mRNAs used in our experiments, Atkins and colleagues made the FSS-destabilizing mutations in the context of a long dnaX/ $\beta$-galactosidase reporter mRNA, which was over 3000 nucleotides long. By computation, we find, in fact, that there are competing base pairs in the construct to the dnaX hairpin pairs (Supplementary Fig. 6). The competing pairs arise from nucleotides just 3' downstream to the $\operatorname{dnaX}$ hairpin and also from nucleotides close to the $3^{\prime}$ end of $\beta$ galactosidase ORF. It is therefore likely that the mutations designed to destabilize the dnaX hairpin resulted in alternative secondary structures, which complicate the analysis of the data.

Our data show that structural properties of the FSS such as stem length and the presence of the loop make significant contribution to FSS-induced long ribosome pauses. Noteworthy, both HIV and dnaX FSSs are 11 basepairs long (Fig. 1b). The idea that optimal stem length (around 11-12 basepairs) is important for ribosome pausing and frameshifting is supported by the study of the frameshift-inducing stem-loop derived from Simian retrovirus type-1 gag-pro transcript $^{62}$. This work demonstrated that both increase and decrease in the length of the stem 
from the original length of 12 basepairs reduce frameshifting efficiency.

How do FSSs interact with the ribosome to inhibit translation elongation? Our recent studies indicated that the FSSs transiently inhibit tRNA biding by directly blocking the A site. In the structure of HIV FSS-ribosome complex, mRNA nucleotides between the P-site codon and the FSS are dissociated from the mRNA tunnel and the FSS is docked into the A site of the ribosome $^{31}$. In this structure, the FSS loop contacts the A-site finger (Helix 38) of 23 rRNA. Consistent with the importance of the FSS loop-ribosome interactions, HIV and dnaX bimolecular helixes lacking the loop do not inhibit A-site tRNA binding. Furthermore, shortening the stem-loop that also likely eliminates the interaction of the loop with the A site finger, and thus partially alleviates the FSS-induced inhibition of A-site tRNA binding. In our experiments, variations in the sequence and length of the loop do not affect the ability of the HIV FSS to inhibit tRNA binding (Fig. 3a). Hence, the interactions between the FSS loop and the large subunit do not appear to be sequence specific and likely involve RNA phosphate backbone. The interactions also seem to play important roles in stabilizing the FSS in the A site.

In addition to inhibiting A-site tRNA binding, the HIV and dnaX FSSs slow down translocation. This likely occurs when mRNA is threaded through the $30 \mathrm{~S}$ mRNA channel and the FSS interacts with the entry to the mRNA tunnel that is lined by ribosomal protein uS3, uS4 and uS5. Such conformation of the FSS was visualized by cryo-EM reconstructions of the dnaX FSS bound to bacterial ribosome ${ }^{42}$ and frameshift-inducing pseudoknot from SARSCoV-2 RNA genome bound to mammalian ribosome ${ }^{63}$. In the dnaX-ribosome complex, similar to dnaX-ribosomes complex used in our work, the FSS was positioned 11 nucleotides 
downstream of the P-site codon ${ }^{42}$. Because of intermediate local resolution at the dnaX hairpin, molecular details of the FSS interactions with E. coli ribosome remain unclear. SHAPE probing of the dnaX FSS performed on this complex demonstrated that dnaX FSS is properly folded when adjacent to mRNA tunnel, although basepairing status of nucleotides $+13,+14$ and +15 of the FSS was ambiguous ${ }^{42,57}$.

Our data support the assumption that when bound at the entry to the mRNA tunnel (i.e. 11 nucleotides downstream of the P-site codon in bacterial ribosome), the HIV and dnaX FSS remain fully folded. Mutations destabilizing basepairing interactions formed by nucleotides +12 , +13 and +14 in the HIV FSS and $+13,+14$ and +15 in the dnaX FSS appreciably affected the rate of translocation in our smFRET experiments indicating that unwinding of these basepairs accompanies translocation (Fig. 5). Ttrl $_{\text {O }}$ observed in the presence of HIV destabilized FSS I (41 s) and HIV destabilized FSS II (32 s) (Fig. 5a) were relatively similar consistent with the idea basepairs formed by nucleotides +15 and +16 are not unwound during translocation in our smFRET experiments.

Similar to earlier proposals ${ }^{63,64}$, we hypothesize that interactions of the FSS with the entry of mRNA tunnel inhibit ribosome translocation and helicase activity. Recent optical tweezers experiments indicated that unwinding of three basepairs adjacent to the mRNA tunnel occurs through two parallel pathways, one of which is 7 -fold slower than the other ${ }^{12}$. An mRNA stemloop likely samples alternative conformations at the entry to mRNA tunnel. In some of these conformations, translocation and basepair unwinding might be slowed down. Unique interactions with the ribosome that distinguish the FSS from the bimolecular helix likely favor this slow translocation/unwinding pathway. Our data show that the presence of the loop and 
specific stem length stabilize the inhibitory conformation of the FSS-ribosome complex. The cryo-EM reconstruction of the SARS-CoV-2 FSS bound at the entry to mRNA tunnel of mammalian ribosome ${ }^{63}$ indicated that the pseudoknot wedges between the head and the body domains of the small ribosomal subunit and thus might restrict the swiveling motion of the head domain relative to the body and platform domains of the small subunit that accompanies ribosome translocation ${ }^{65}$. Structures with higher local resolution near the FSS positioned at entry to the mRNA tunnel will be required to elucidate the molecular mechanism of FSSmediated switching between the slow and fast pathways of translocation/mRNA unwinding observed in optical tweezers experiments.

Interestingly, when positioned 11 nucleotides downstream of the P-site codon, a bimolecular helix identical to the stem of the HIV FSS, inhibits translocation less than the wildtype HIV FSS positioned 12 or 13 nucleotides downstream of the P-site codon. In other words, unwinding of three basepairs of the HIV bimolecular helix slows down the ribosome less than unwinding of two basepairs or one basepair of the HIV FSS. This observation is consistent with the interpretation that thermodynamic stabilities of basepairs adjacent to the entry to the mRNA tunnel do not account for the inhibitory effect of the FSS. Partial alleviation of the FSSmediated pausing observed in our experiments when the spacing between the FSS and the P-site codon was extended from 11 to 12 or 13 nucleotides likely indicates that the short (11 nucleotide) spacer provides entropic stabilization for the inhibitory interactions between the FSS and the ribosome by restricting the FSS dynamics.

Our findings have a number of implications for translational regulation at transcriptomewide levels. On the one hand, because unwinding of three basepairs slows down the ribosome 
only 2-3 times, most elements of secondary structure are not expected to produce long ribosome pauses that are consistent with published ribosome profiling studies ${ }^{14,15}$. However, variations in the propensity to form basepairing interactions within the ORF may fine-tune the elongation rate and thus, at least to some extent, regulate the translational efficiency ${ }^{16}$.

On the other hand, mRNA stem-loops whose specific stem length and loop structure enable them to either dock into the A site to block tRNA binding or to interact with the entry to mRNA channel to hinder translocation can produce extended ribosome pauses. These pauses might be more transient in live cells than the pauses observed in our in vitro experiments because combining the FSS with the non-slippery sequences may exacerbate the FSS-induced inhibition of translation $^{18,31}$. Furthermore, cellular concentrations of EF-Tu and EF-G might be higher than those used in our experiments. Nevertheless, our experiments provide several lines of evidence that specific mRNA stem-loops inhibit translation much stronger than an mRNA helix in general. Further investigation of structural properties of mRNA stem-loops and pseudoknots important for translation inhibition may inform identification of previously unknown regulatory secondary structure elements within mRNA ORFs that produce programmed ribosome pausing events.

\section{Methods}

\section{Preparation of ribosome, translation factors, tRNA and mRNA}

The tight couple $70 S$ ribosomes were purified from E. coli MRE600 strain ${ }^{47}$. S6-cy5/L9-cy3 ribosomes were prepared by partial reconstitution of $\Delta S 6-30 S$ and $\Delta L 9-50 S$ subunits with S641C-cy5 and L11-11C-cy3 proteins ${ }^{47,66}$. tRNAs (purchased from Chemical Block) were aminocylated as previously described ${ }^{53}$. Histidine-tagged EF-G and EF-Tu were expressed 
and purified by standard procedures. Sequences encoding dnaX and HIV mRNA variants (Supplementary table 1) were cloned and prepared using T7 polymerase and run-off in vitro transcription ${ }^{31}$. The bimolecular RNA helices were prepared by annealing $3 \mu \mathrm{M}$ mRNA with 6 $\mu \mathrm{M}$ complementary RNA oligo (synthesized by IDT, Supplementary table 1 ) at $65^{\circ} \mathrm{C}$ for 3 min in polyamine buffer (50 mM HEPES ( $\mathrm{pH} 7.5), 6 \mathrm{mM} \mathrm{Mg}^{2+}, 6 \mathrm{mM} \beta$-mercaptoethanol, $150 \mathrm{mM}$ $\mathrm{NH}_{4} \mathrm{Cl}, 0.1 \mathrm{mM}$ spermine and $2 \mathrm{mM}$ spermidine).

\section{smFRET measurements}

The total internal reflection fluorescence (TIRF) microscopy-based smFRET experiments were performed using published procedures ${ }^{31,48,66}$ with modifications. Quartz slides were coated with dichlorodimethylsilane (DDS), bound with biotin-BSA and passivated by Tween$20^{67} .30 \mu \mathrm{L} 0.2 \mathrm{mg} / \mathrm{mL}$ neutravidin dissolved in H50 buffer (20 mM HEPES (pH 7.5) and 50 $\mathrm{mM} \mathrm{KCl)}$ was loaded and bound to the biotin-BSA. Ribosomal complexes were imaged in polyamine buffer (50 mM HEPES ( $\mathrm{pH} 7.5), 6 \mathrm{mM} \mathrm{Mg}{ }^{2+}, 6 \mathrm{mM} \beta$-mercaptoethanol, $150 \mathrm{mM}$ $\mathrm{NH}_{4} \mathrm{Cl}, 0.1 \mathrm{mM}$ spermine and $2 \mathrm{mM}$ spermidine) with $0.8 \mathrm{mg} / \mathrm{mL}$ glucose oxidase, $0.625 \%$ glucose, $0.4 \mu \mathrm{g} / \mathrm{mL}$ catalase and 1.5 mM 6-hydroxy-2,5,7,8-tetramethylchromane-2-carboxylic (Trolox). The ribosome complexes containing bimolecular RNA helices were imaged in the presence $1 \mu \mathrm{M}$ complementary RNA oligo to assure oligo re-annealing. FRET data were acquired and processed as previously described ${ }^{31,66}$. Rates of fluctuations between 0.4 and 0.6 FRET states were determined by idealizing FRET traces with 2-state Hidden Markov model $(\mathrm{HMM})^{68}$.

Ribosome complexes used in smFRET experiments were assembled as follows. $0.3 \mu \mathrm{M}$ S6/L9-labeled ribosomes were incubated with $0.6 \mu \mathrm{M}$ tRNA and $0.6 \mu \mathrm{M}$ mRNA in polyamine 
buffer at $37^{\circ} \mathrm{C}$ for $15 \mathrm{~min}$. To fill the A site, $0.6 \mu \mathrm{M}$ aminoacyl-tRNA were pre-incubated with 10 $\mu \mathrm{M}$ EF-Tu and $1 \mathrm{mM}$ GTP in polyamine buffer at $37^{\circ} \mathrm{C}$ for $10 \mathrm{~min}$. To overcome the FSSinduced inhibition of tRNA binding, $0.3 \mu \mathrm{M}$ ribosomal complex containing peptidyl-tRNA in the P site was incubated with $0.6 \mu \mathrm{M}$ aminoacyl-tRNA (complexed with EF-Tu・GTP) at $37^{\circ} \mathrm{C}$ for $10 \mathrm{~min}$. The ribosome samples were loaded and immobilized on the slide as previously described $^{31}$. To catalyze translocation, $1 \mu \mathrm{M}$ EF-G•GTP (in imaging buffer) was delivered at $0.4 \mathrm{~mL} / \mathrm{min}$ speed by syringe pump at $10 \mathrm{~s}$ of imaging.

\section{Filter-binding assay}

Filter-binding assays were performed using standard procedures ${ }^{31,69}$ with minor modifications. Ribosome complexes were assembled and incubated with aminoacyl-tRNA charged with radio-labeled amino acid as described previously ${ }^{31}$. Similar to the smFRET experiments described above, the complexes containing the bimolecular RNA helices were incubated in the presence of $1 \mu \mathrm{M}$ complementary RNA oligo. The ribosome complexes were applied to a nitrocellulose filter (MiliporeSigma), which was immediately washed with $500 \mu \mathrm{l}$ (for dnaXribosome complexes) or $800 \mu \mathrm{l}$ (for HIV- or m291-ribosome complexes) of ice-cold polyamine buffer containing $20 \mathrm{mM} \mathrm{Mg}^{2+}$. The $20 \mathrm{mM} \mathrm{Mg}^{2+}$ concentration was used to stabilize the ribosome complexes under non-equilibrium condition.

\section{RNAstructure prediction}

RNAstructure (version 6.2) was used to prediction the RNA secondary structure and thermodynamic stability of corresponding structures ${ }^{45}$. RNA secondary structure were predicted using maximum expected accuracy ${ }^{70}$ from basepair probabilities estimated from a 
partition function calculation. In these calculations, nucleotides from the beginning of the Shine-Dalgarno sequence to nucleotide +11 downstream of the first nucleotide of the P-site codon and the oligonucleotide tether binding site were forced to be unpaired, mimicking the effect of the bound ribosome and oligonucleotide. The efn2 function is used to calculate the $\Delta \mathrm{G}^{\circ}$ of RNA secondary structure based on the predicted structure. For the bimolecular structure prediction, bifold was used ${ }^{71}$.

ACKNOWLEDGEMENTS. These studies were supported by grant from the US National Institute of Health R01GM099719 (to D.N.E.) and R01GM076485 (to D.H.M.). We thank Harry Noller, Laura Lancaster and Gillian Rexroad for their comments on the manuscript.

\section{AUTHOR CONTRIBUTIONS}

D.N.E. conceived the project. C.B., M.Z., D.H.M. and D.N.E. designed research. C.B. performed experiments with contributions from I.N. and H.W. M.Z. performed computational studies. C.B., M.K., D.H.M. and D.N.E. wrote the paper. 


\section{Figures}

a

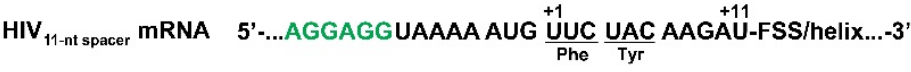

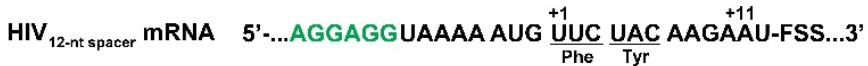

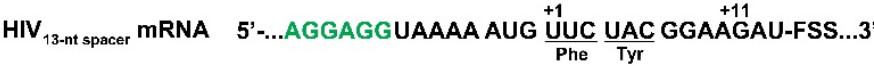

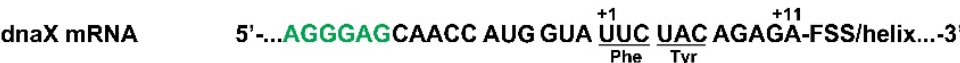

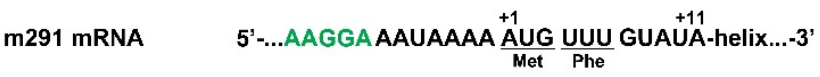

b

$\Delta \mathbf{G}^{\circ}(\mathrm{kcal} / \mathrm{mol})$

$\Delta \Delta \mathbf{G}^{\circ}(\mathrm{kcal} / \mathrm{mol})$
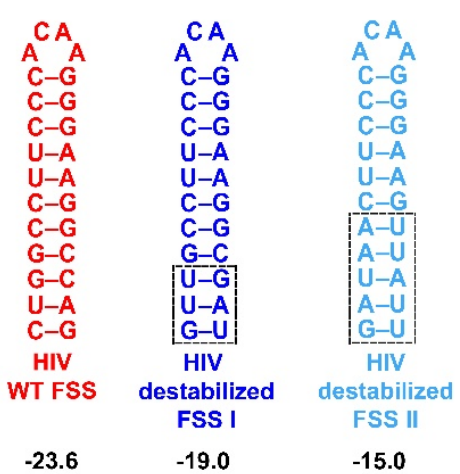

$-23.6$
$-19.0$

4.6

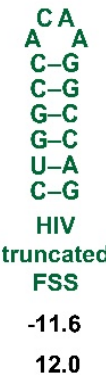

c

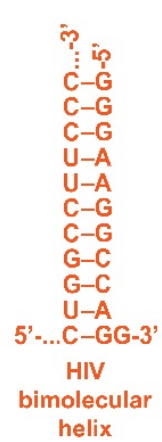

$\Delta \mathbf{G}^{\circ}(\mathrm{kcal} / \mathrm{mol})$

$-25.3$

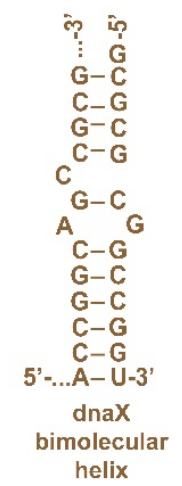

$-20.3$

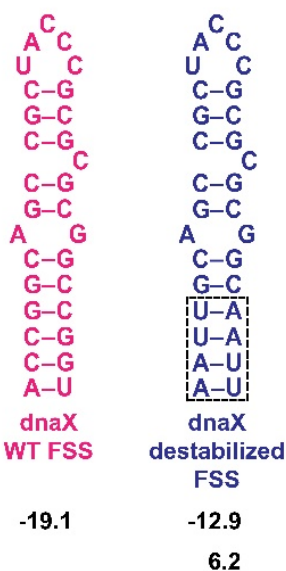

$-0.1$

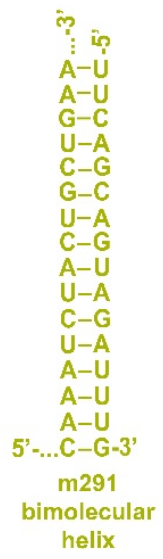

$-24.5$

Figure 1. Model mRNAs containing either an FSS stem-loop or a bimolecular helix.

(A) Sequences of model mRNAs containing the Shine-Dalgarno (SD) sequences (colored green) and the FSS. The first nucleotide of P-site codon and the last nucleotide bound in mRNA tunnel are labeled +1 and +11 , respectively. (B-C) Secondary structures of frameshiftstimulating stem-loops (B) or bimolecular RNA helices (C). Dashed boxes indicate replacements of native $G C$ basepairs with less stable $A-U$ or $G-U$ pairs. $\Delta G$ values represent the overall thermodynamic stability of corresponding structures. $\Delta \Delta G^{\circ}$ values of FSS variants indicate the changes in stabilities relative to the corresponding WT hairpins. Secondary structures and $\Delta G^{\circ} / \Delta \Delta G^{\circ}$ values (B-C) were determined by the RNAstructure software ${ }^{45}$. 


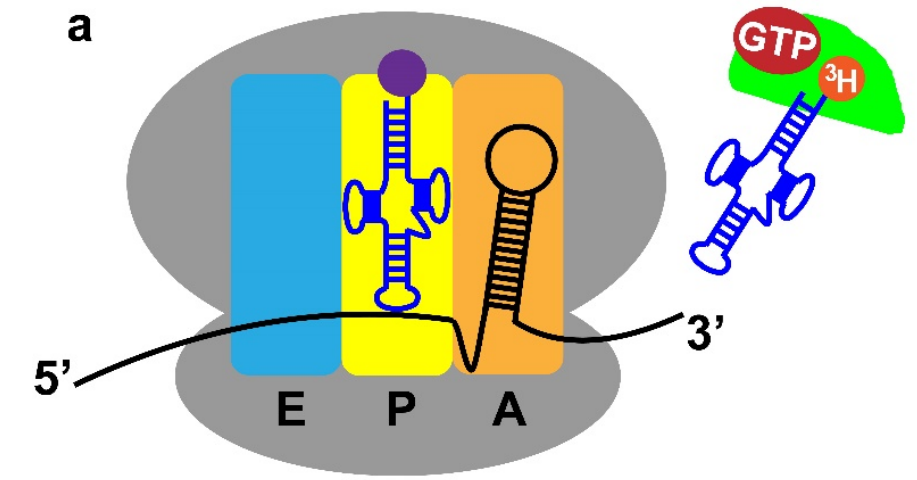

FSS-mediated inhibition of A-site tRNA binding

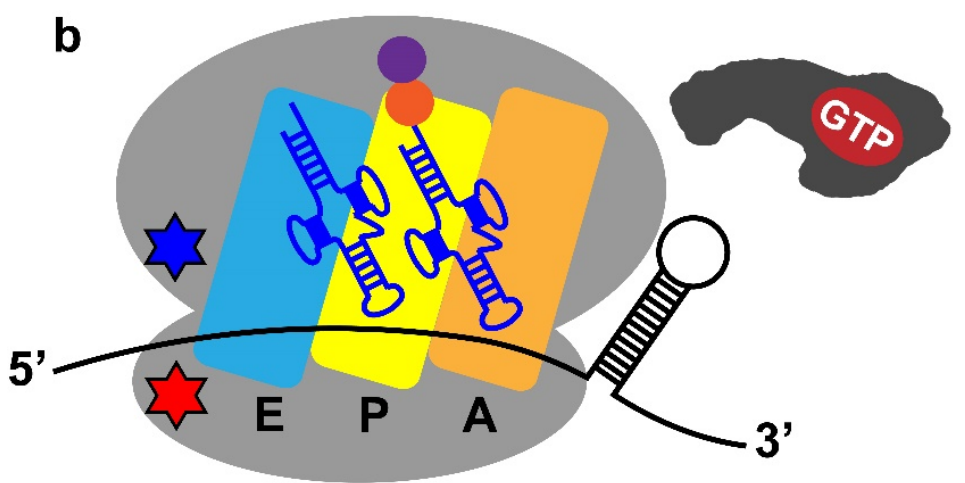

FSS-mediated inhibition of tRNA/mRNA translocation

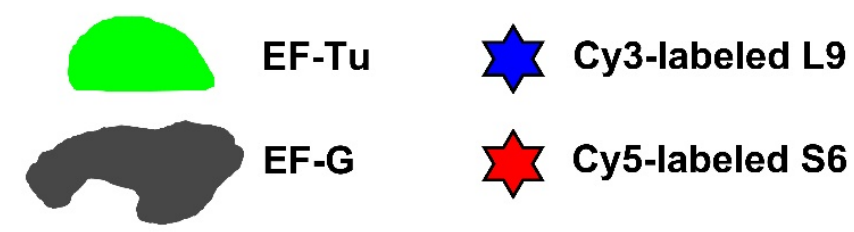

Figure 2. Experimental design

(a) The effects of FSS variants on A-site tRNA binding were determined by filter-binding assays. $70 \mathrm{~S}$ bacterial ribosome (grey) bound with P-site peptidyltRNA and mRNA was incubated with aminoacyl-tRNA, which had been charged with tritium-labeled amino acid and complexed with EF-Tu•GTP. (b) The effects of FSS variants on ribosome translocation coupled to intersubunit rotation was studied by smFRET. Cy5 (red hexagram) and cy3 (blue hexagram) fluorophores were attached to the $30 S$ protein S6 and 50S protein L9, respectively. The pretranslocation S6-cy5/L9-cy3labeled ribosome contained deacylated and peptidyl-tRNAs that adoped P/E and A/P hybrid states in the $R$ conformation of the ribosome. EF-G.GTP was delivered to the ribosome by injection. The amino acids bonded with cognate tRNAs are shown in purple and orange. $A, P$ and $E$ sites of the ribosome are indicated by orange, yellow and blue, respectively. 


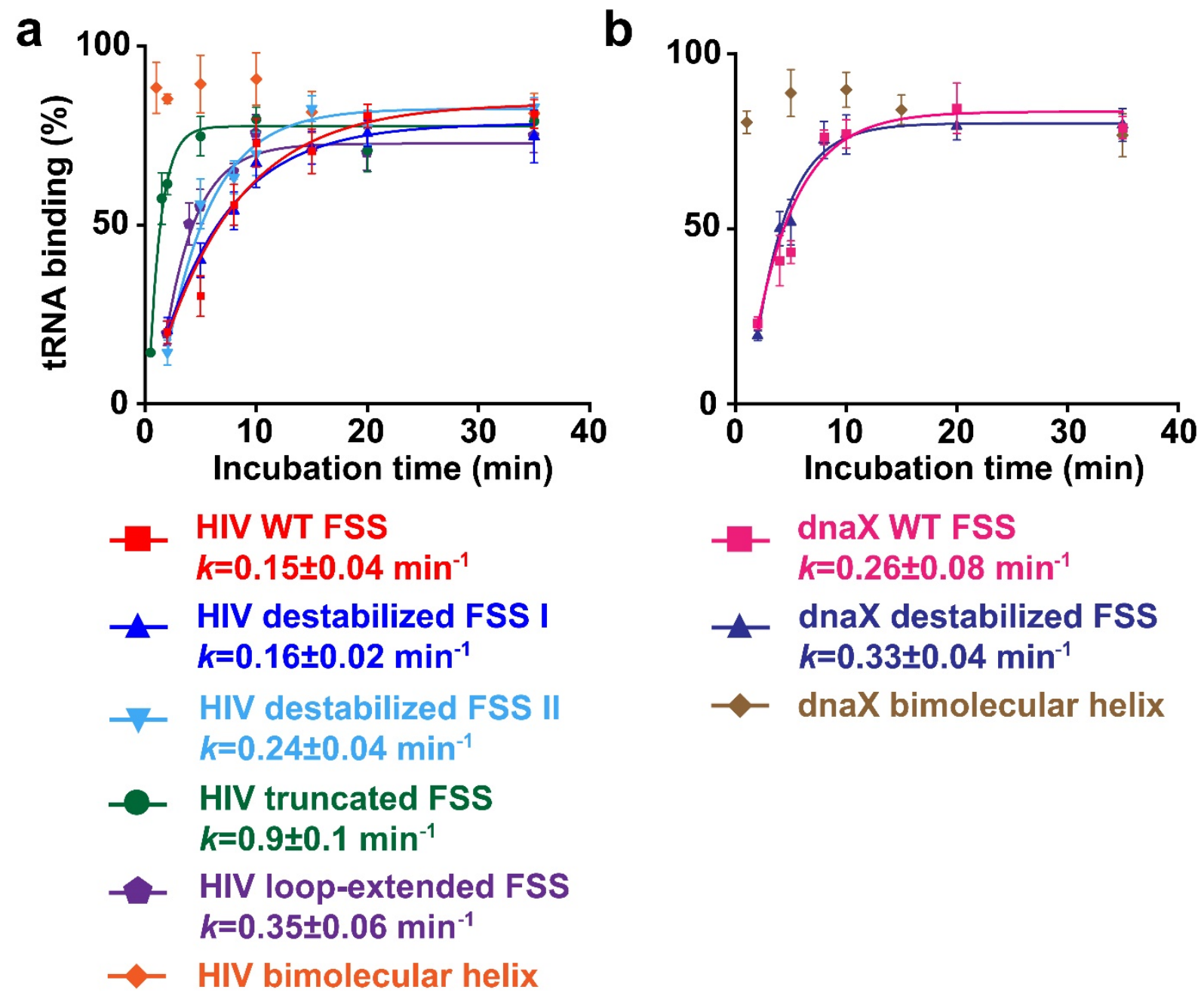

Figure 3. The stem length and the presence of the loop are more important for the FSS-induced inhibition of tRNA binding than FSS local and overall thermodynamic stabilities.

Kinetics of EF-Tu-catalyzed $\left[{ }^{3} \mathrm{H}\right]$ Tyr-tRNA ${ }^{\text {Tyr }}$ binding to the A site of the $70 \mathrm{~S}$ ribosome. The ribosomes contained $N$-Ac-Phe-tRNA ${ }^{\text {Phe }}$ in the $\mathrm{P}$ site and were programmed with HIV (a) or dnaX (b) mRNAs, which contained an FSS variant (Figure 1) 11 nucleotides downstream of the P-site codon as indicated. The binding of radio-labeled $\left[{ }^{3} \mathrm{H}\right]$ Tyr-tRNA ${ }^{\mathrm{Tyr}}$ to ribosomes programmed with FSS-containing mRNA is shown relative to that observed in ribosomes programmed with corresponding $\triangle \mathrm{FSS}$ mRNA. Apparent pseudo first order tRNA binding rates were deduced from single-exponential fitting as shown by the line graphs. 


\section{a}

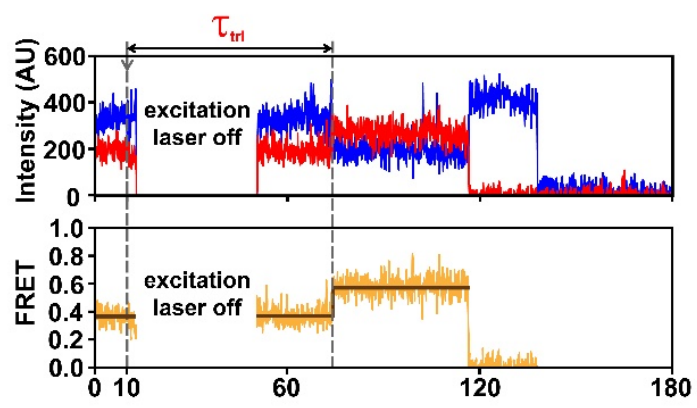

c
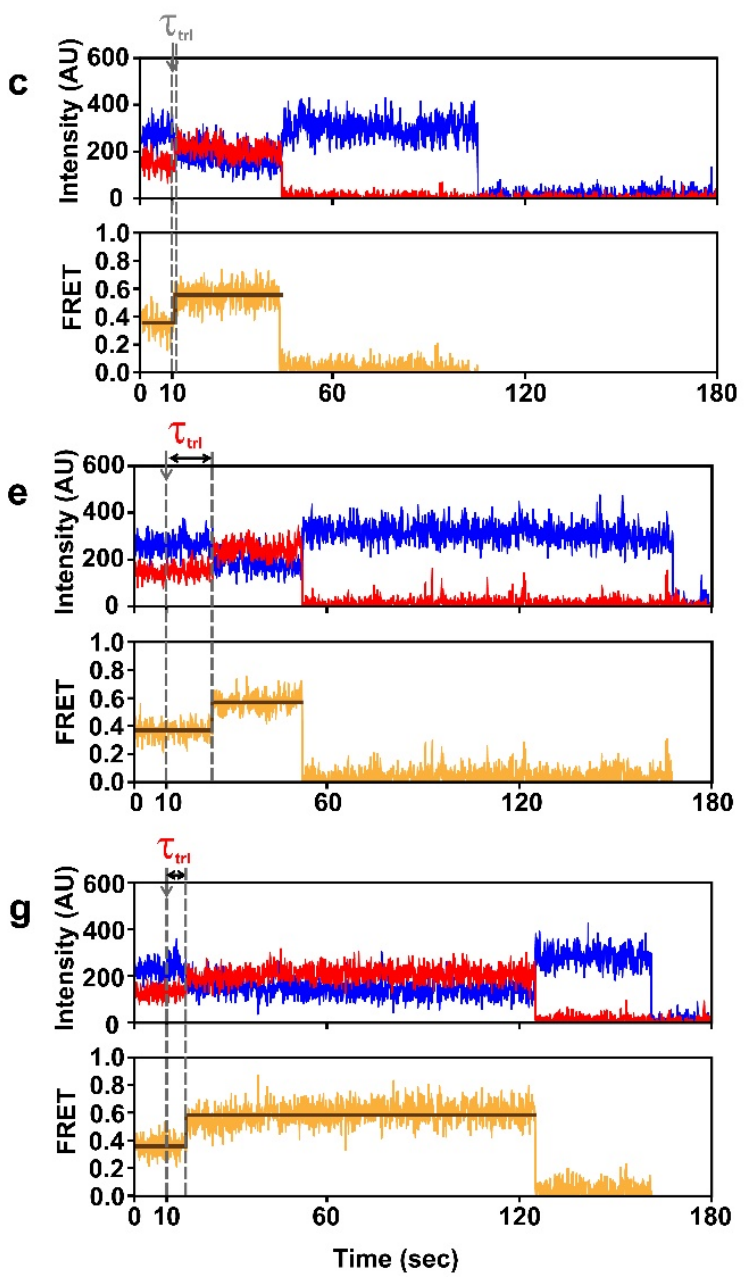

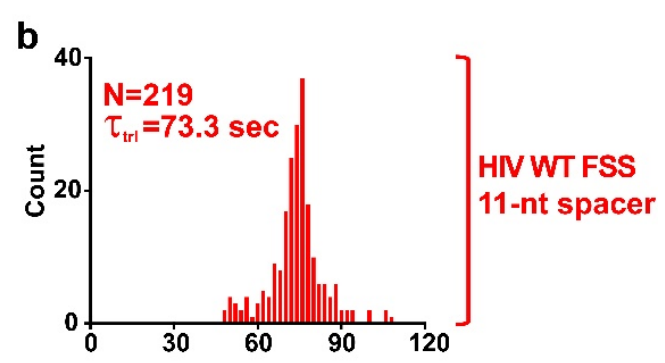

d

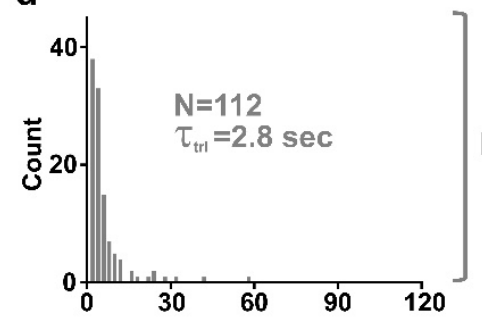

HIV $\Delta$ FSS

f

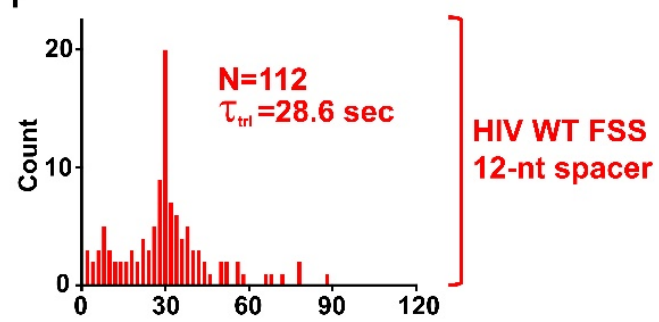

h

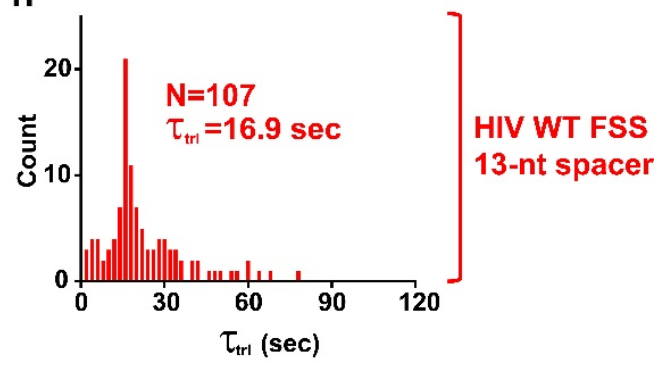

Figure 4. The FSS-induced inhibition of translocation is influenced by length of the mRNA spacer between P-site codon and FSS.

The pre-translocation S6-cy5/L9-cy3-labeled ribosomes were programmed with either HIV WT FSS (a, b, e, f, g, h) or HIV $\triangle F S S$ (c, d) mRNA. The HIV FSS was positioned either 11 (a, b), $12(\mathbf{e}, \mathbf{f})$ or $13(\mathbf{g}, \mathbf{h})$ nucleotides downstream of the P-site codon, respectively. $(\mathbf{a}, \mathbf{c}, \mathbf{e}, \mathbf{g})$ Representative FRET traces show cy3 donor fluorescence (blue), cy5 acceptor fluorescence (red) and FRET efficiency (orange) fitted by two-state HHM (brown). Arrows show the injection of EF-G-GTP. The EF-G-catalyzed translocation corresponds to the transition from R $(0.4$ FRET) to a stable (i.e. lasting over $4 \mathrm{~s}$ ) NR (0.6 FRET) state of the ribosome. (b, d, $\mathbf{f}, \mathbf{h}$ ) Histograms ( $2 \mathrm{~s}$ binning size) show the distributions and median values of represents the dwell time between the injection and the translocation, $T_{\text {trl }} . N$ indicates the number of traces assembled into each histogram. To extend lifetime of the acceptor fluorophore in the 
experiments with 11 nucleotide spacer mRNA, the excitation laser was turned off after the EF$\mathrm{G}$ injection and switched back on either $40 \mathrm{~s}$ (a) or $60 \mathrm{~s}$ later. Ttrl distribution in (b) was combined from data sets acquired with 40s (Supplementary Fig. 3a) and $60 \mathrm{~s}$ (Supplementary Fig. 3b) laser shut-off times.

a

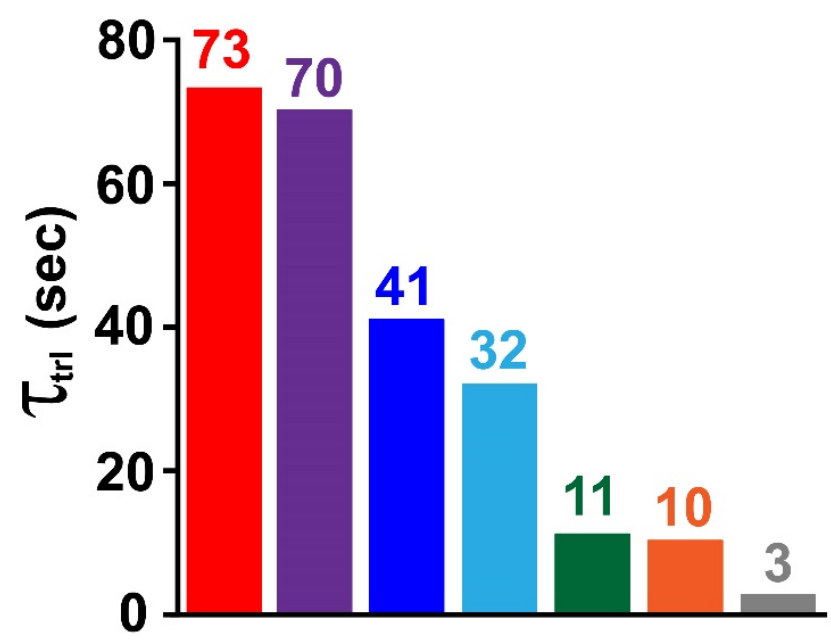

HIV WT FSS

HIV loop-extended FSS

HIV destabilized FSS I

HIV destabilized FSS II

HIV truncated FSS

HIV bimolecular helix

HIV $\triangle F S S$ b

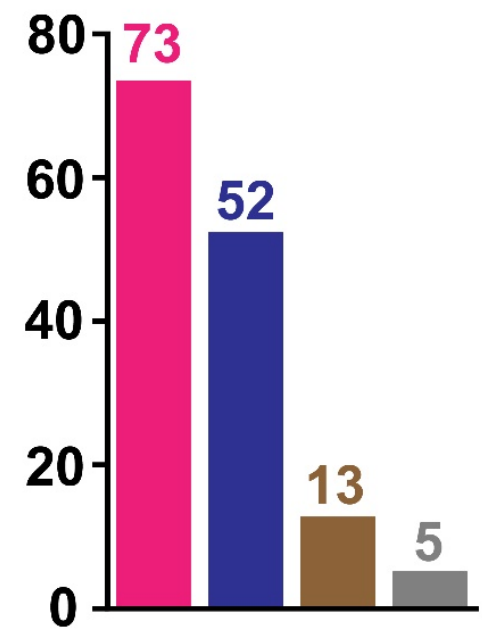

dnaX WT FSS dnaX destabilized FSS dnaX bimolecular helix dnaX $\triangle$ FSS

Figure 5. The stem length and the presence of the loop are more important for the FSSinduced inhibition of translocation than FSS local and overall thermodynamic stabilities.

The pre-translocation S6-cy5/L9-cy3 ribosomes were programmed with mRNA containing either a HIV (a) or dnaX (b) FSS variants as indicated. Bar graphs show the median values of $T_{\text {trl }}$, which represents the dwell time between injection of EF-G•GTP and ribosome translocation in smFRET experiments. 

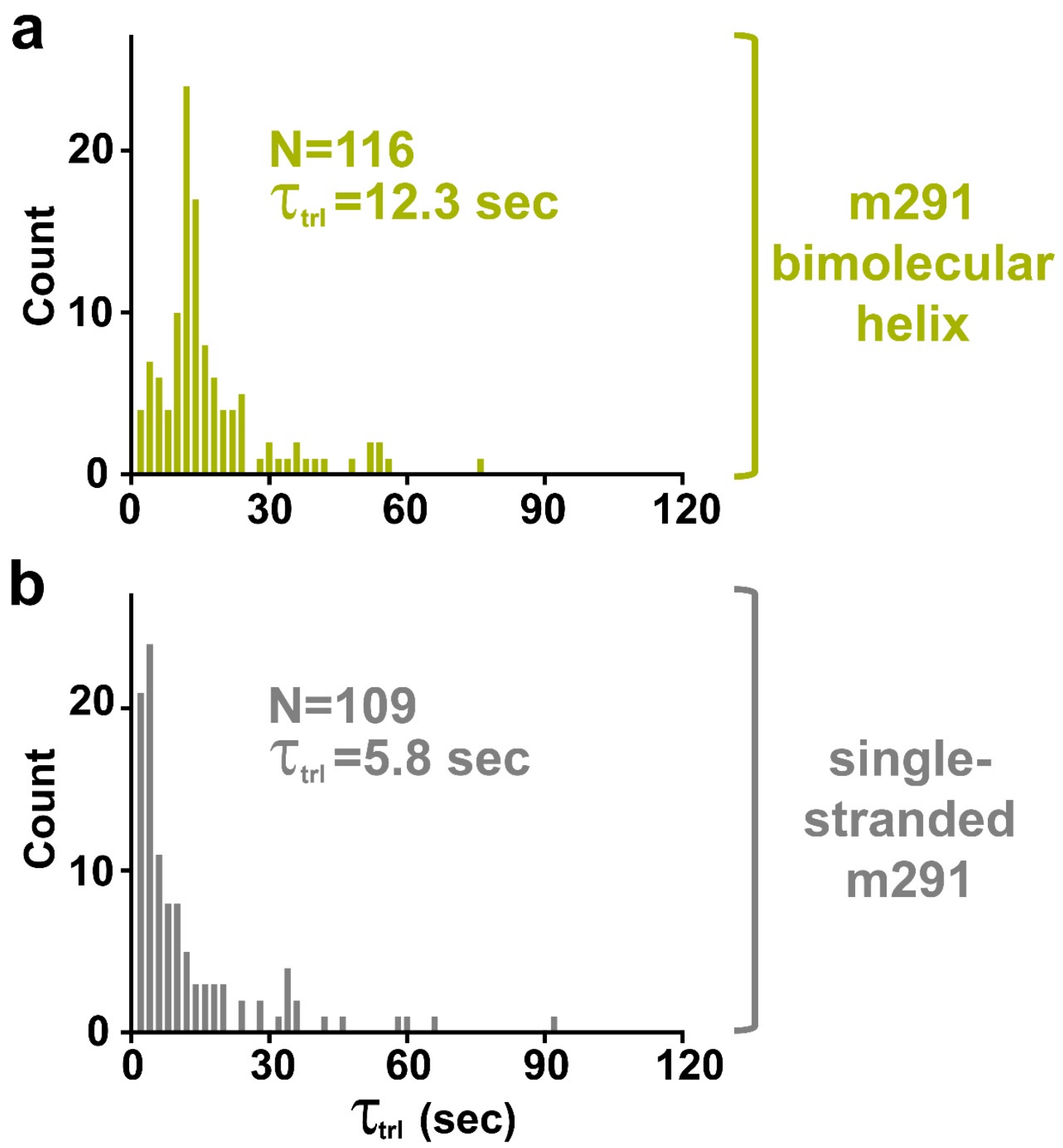

Figure 6. The bimolecular helix formed in the m291 mRNA at the entry to mRNA tunnel mildly inhibits translocation.

Kinetics of translocation was measured by smFRET experiments with pre-translocation S6cy5/L9-cy3 ribosomes programmed with m291 mRNA containing a 16-bp bimolecular RNA helix positioned 11 nucleotides downstream of P-site codon (a) or with single-stranded $\mathrm{m} 291$ mRNA (b). Histograms (2 s binning size) show the distributions and median values of $\mathrm{T}_{\mathrm{tr}}$, which represents the dwell time between injection of EF-G.GTP and ribosome translocation. $\mathrm{N}$ indicates the number of FRET traces compiled into each histogram. 


\section{References}

1. Yusupova, G.Z., Yusupov, M.M., Cate, J.H. \& Noller, H.F. The path of messenger RNA through the ribosome. Cell 106, 233-41 (2001).

2. Yusupova, G., Jenner, L., Rees, B., Moras, D. \& Yusupov, M. Structural basis for messenger RNA movement on the ribosome. Nature 444, 391-4 (2006).

3. Jenner, L.B., Demeshkina, N., Yusupova, G. \& Yusupov, M. Structural aspects of messenger RNA reading frame maintenance by the ribosome. Nat Struct Mol Biol 17, 555-60 (2010).

4. Takyar, S., Hickerson, R.P. \& Noller, H.F. mRNA helicase activity of the ribosome. Cell 120, 49-58 (2005).

5. Wen, J.D. et al. Following translation by single ribosomes one codon at a time. Nature 452, 598-603 (2008).

6. Adivarahan, S. et al. Spatial Organization of Single mRNPs at Different Stages of the Gene Expression Pathway. Mol Cell 72, 727-738 e5 (2018).

7. Khong, A. \& Parker, R. mRNP architecture in translating and stress conditions reveals an ordered pathway of mRNP compaction. J Cell Biol 217, 4124-4140 (2018).

8. Aw, J.G. et al. In Vivo Mapping of Eukaryotic RNA Interactomes Reveals Principles of Higher-Order Organization and Regulation. Mol Cell 62, 603-17 (2016).

9. Lu, Z. et al. RNA Duplex Map in Living Cells Reveals Higher-Order Transcriptome Structure. Cell 165, 1267-1279 (2016).

10. Qu, X. et al. The ribosome uses two active mechanisms to unwind messenger RNA during translation. Nature 475, 118-21 (2011).

11. Caliskan, N., Katunin, V.I., Belardinelli, R., Peske, F. \& Rodnina, M.V. Programmed -1 frameshifting by kinetic partitioning during impeded translocation. Cell 157, 1619-31 (2014).

12. Desai, V.P. et al. Co-temporal Force and Fluorescence Measurements Reveal a Ribosomal Gear Shift Mechanism of Translation Regulation by Structured mRNAs. Mol Cell 75, 1007-1019 e5 (2019).

13. Chen, C. et al. Dynamics of translation by single ribosomes through mRNA secondary structures. Nat Struct Mol Biol 20, 582-8 (2013).

14. Del Campo, C., Bartholomaus, A., Fedyunin, I. \& Ignatova, Z. Secondary Structure across the Bacterial Transcriptome Reveals Versatile Roles in mRNA Regulation and Function. PLoS Genet 11, e1005613 (2015).

15. Pop, C. et al. Causal signals between codon bias, mRNA structure, and the efficiency of translation and elongation. Mol Syst Biol 10, 770 (2014).

16. Burkhardt, D.H. et al. Operon mRNAs are organized into ORF-centric structures that predict translation efficiency. Elife 6(2017).

17. Gorochowski, T.E., Ignatova, Z., Bovenberg, R.A. \& Roubos, J.A. Trade-offs between tRNA abundance and mRNA secondary structure support smoothing of translation elongation rate. Nucleic Acids Res 43, 3022-32 (2015).

18. Yan, S., Wen, J.D., Bustamante, C. \& Tinoco, I., Jr. Ribosome excursions during mRNA 
translocation mediate broad branching of frameshift pathways. Cell 160, 870-81 (2015).

19. Doma, M.K. \& Parker, R. Endonucleolytic cleavage of eukaryotic mRNAs with stalls in translation elongation. Nature 440, 561-4 (2006).

20. Young, J.C. \& Andrews, D.W. The signal recognition particle receptor alpha subunit assembles co-translationally on the endoplasmic reticulum membrane during an mRNA-encoded translation pause in vitro. EMBO J 15, 172-81 (1996).

21. Caliskan, N., Peske, F. \& Rodnina, M.V. Changed in translation: mRNA recoding by -1 programmed ribosomal frameshifting. Trends Biochem Sci 40, 265-74 (2015).

22. Jacks, T. et al. Characterization of ribosomal frameshifting in HIV-1 gag-pol expression. Nature 331, 280-3 (1988).

23. Kelly, J.A. et al. Structural and functional conservation of the programmed -1 ribosomal frameshift signal of SARS coronavirus 2 (SARS-CoV-2). J Biol Chem 295, 1074110748 (2020).

24. Kelly, J.A., Woodside, M.T. \& Dinman, J.D. Programmed -1 Ribosomal Frameshifting in coronaviruses: A therapeutic target. Virology 554, 75-82 (2021).

25. Atkins, J.F. et al. Overriding standard decoding: implications of recoding for ribosome function and enrichment of gene expression. Cold Spring Harb Symp Quant Biol 66, 217-32 (2001).

26. Bock, L.V. et al. Thermodynamic control of -1 programmed ribosomal frameshifting. Nat Commun 10, 4598 (2019).

27. Chen, J. et al. Dynamic pathways of -1 translational frameshifting. Nature 512, 328-32 (2014).

28. Kim, H.K. et al. A frameshifting stimulatory stem loop destabilizes the hybrid state and impedes ribosomal translocation. Proc Natl Acad Sci U S A 111, 5538-43 (2014).

29. Caliskan, N. et al. Conditional Switch between Frameshifting Regimes upon Translation of dnaX mRNA. Mol Cell 66, 558-567 e4 (2017).

30. Choi, J., O'Loughlin, S., Atkins, J.F. \& Puglisi, J.D. The energy landscape of -1 ribosomal frameshifting. Sci Adv 6, eaax6969 (2020).

31. Bao, C. et al. mRNA stem-loops can pause the ribosome by hindering A-site tRNA binding. Elife 9(2020).

32. Larsen, B., Gesteland, R.F. \& Atkins, J.F. Structural probing and mutagenic analysis of the stem-loop required for Escherichia coli dnaX ribosomal frameshifting: programmed efficiency of 50\%. J Mol Biol 271, 47-60 (1997).

33. Dinman, J.D. Mechanisms and implications of programmed translational frameshifting. Wiley Interdiscip Rev RNA 3, 661-73 (2012).

34. Mouzakis, K.D., Lang, A.L., Vander Meulen, K.A., Easterday, P.D. \& Butcher, S.E. HIV1 frameshift efficiency is primarily determined by the stability of base pairs positioned at the mRNA entrance channel of the ribosome. Nucleic Acids Res 41, 1901-13 (2013).

35. Garcia-Miranda, P. et al. HIV frameshift site RNA stability correlates with frameshift efficiency and decreased viral infectivity. J Virol (2016).

36. Spasic, A., Assmann, S.M., Bevilacqua, P.C. \& Mathews, D.H. Modeling RNA secondary structure folding ensembles using SHAPE mapping data. Nucleic Acids Res 46, 314-323 (2018).

37. Kim, H.K. \& Tinoco, I., Jr. EF-G catalyzed translocation dynamics in the presence of 
ribosomal frameshifting stimulatory signals. Nucleic Acids Res 45, 2865-2874 (2017).

38. Brunelle, M.N., Payant, C., Lemay, G. \& Brakier-Gingras, L. Expression of the human immunodeficiency virus frameshift signal in a bacterial cell-free system: influence of an interaction between the ribosome and a stem-loop structure downstream from the slippery site. Nucleic Acids Res 27, 4783-91 (1999).

39. Leger, M., Sidani, S. \& Brakier-Gingras, L. A reassessment of the response of the bacterial ribosome to the frameshift stimulatory signal of the human immunodeficiency virus type 1. RNA 10, 1225-35 (2004).

40. Mazauric, M.H., Seol, Y., Yoshizawa, S., Visscher, K. \& Fourmy, D. Interaction of the HIV-1 frameshift signal with the ribosome. Nucleic Acids Res 37, 7654-64 (2009).

41. Korniy, N. et al. Modulation of HIV-1 Gag/Gag-Pol frameshifting by tRNA abundance. Nucleic Acids Res 47, 5210-5222 (2019).

42. Zhang, Y., Hong, S., Ruangprasert, A., Skiniotis, G. \& Dunham, C.M. Alternative Mode of E-Site tRNA Binding in the Presence of a Downstream mRNA Stem Loop at the Entrance Channel. Structure 26, 437-445 e3 (2018).

43. Qin, P., Yu, D., Zuo, X. \& Cornish, P.V. Structured mRNA induces the ribosome into a hyper-rotated state. EMBO Rep 15, 185-90 (2014).

44. Tsuchihashi, Z. \& Brown, P.O. Sequence requirements for efficient translational frameshifting in the Escherichia coli dnaX gene and the role of an unstable interaction between tRNA(Lys) and an AAG lysine codon. Genes Dev 6, 511-9 (1992).

45. Reuter, J.S. \& Mathews, D.H. RNAstructure: software for RNA secondary structure prediction and analysis. BMC Bioinformatics 11, 129 (2010).

46. Bloomfield, V.A., Crothers, D.M. \& Tinoco, I. Nucleic acids : structures, properties, and functions, x, 794 p. (University Science Books, Sausalito, Calif., 2000).

47. Ermolenko, D.N. et al. Observation of intersubunit movement of the ribosome in solution using FRET. J Mol Biol 370, 530-40 (2007).

48. Cornish, P.V., Ermolenko, D.N., Noller, H.F. \& Ha, T. Spontaneous intersubunit rotation in single ribosomes. Mol Cell 30, 578-88 (2008).

49. Aitken, C.E. \& Puglisi, J.D. Following the intersubunit conformation of the ribosome during translation in real time. Nat Struct Mol Biol 17, 793-800 (2010).

50. Frank, J. \& Gonzalez, R.L., Jr. Structure and dynamics of a processive Brownian motor: the translating ribosome. Annu Rev Biochem 79, 381-412 (2010).

51. Frank, J. \& Agrawal, R.K. A ratchet-like inter-subunit reorganization of the ribosome during translocation. Nature 406, 318-22 (2000).

52. Dunkle, J.A. et al. Structures of the bacterial ribosome in classical and hybrid states of tRNA binding. Science 332, 981-4 (2011).

53. Moazed, D. \& Noller, H.F. Intermediate states in the movement of transfer RNA in the ribosome. Nature 342, 142-8 (1989).

54. Blanchard, S.C., Kim, H.D., Gonzalez, R.L., Jr., Puglisi, J.D. \& Chu, S. tRNA dynamics on the ribosome during translation. Proc Natl Acad Sci U S A 101, 12893-8 (2004).

55. Valle, M. et al. Locking and unlocking of ribosomal motions. Cell 114, 123-34 (2003).

56. Ermolenko, D.N. \& Noller, H.F. mRNA translocation occurs during the second step of ribosomal intersubunit rotation. Nat Struct Mol Biol 18, 457-62 (2011).

57. Amiri, H. \& Noller, H.F. Structural evidence for product stabilization by the ribosomal 
mRNA helicase. RNA 25, 364-375 (2019).

58. Fei, J., Kosuri, P., MacDougall, D.D. \& Gonzalez, R.L., Jr. Coupling of ribosomal L1 stalk and tRNA dynamics during translation elongation. Mol Cell 30, 348-59 (2008).

59. Fredrick, K. \& Noller, H.F. Accurate translocation of mRNA by the ribosome requires a peptidyl group or its analog on the tRNA moving into the 30S P site. Mol Cell 9, 112531 (2002).

60. Ritchie, D.B., Foster, D.A. \& Woodside, M.T. Programmed -1 frameshifting efficiency correlates with RNA pseudoknot conformational plasticity, not resistance to mechanical unfolding. Proc Natl Acad Sci U S A 109, 16167-72 (2012).

61. Wu, B. et al. Translocation kinetics and structural dynamics of ribosomes are modulated by the conformational plasticity of downstream pseudoknots. Nucleic Acids Res 46, 9736-9748 (2018).

62. Yu, C.H., Noteborn, M.H., Pleij, C.W. \& Olsthoorn, R.C. Stem-loop structures can effectively substitute for an RNA pseudoknot in -1 ribosomal frameshifting. Nucleic Acids Res 39, 8952-9 (2011).

63. Bhatt, P.R. et al. Structural basis of ribosomal frameshifting during translation of the SARS-CoV-2 RNA genome. Science 372, 1306-1313 (2021).

64. Namy, O., Moran, S.J., Stuart, D.I., Gilbert, R.J. \& Brierley, I. A mechanical explanation of RNA pseudoknot function in programmed ribosomal frameshifting. Nature 441, 2447 (2006).

65. Noller, H.F., Lancaster, L., Zhou, J. \& Mohan, S. The ribosome moves: RNA mechanics and translocation. Nat Struct Mol Biol 24, 1021-1027 (2017).

66. Ling, C. \& Ermolenko, D.N. Initiation factor 2 stabilizes the ribosome in a semirotated conformation. Proc Natl Acad Sci U S A 112, 15874-9 (2015).

67. Hua, B. et al. An improved surface passivation method for single-molecule studies. Nat Methods 11, 1233-6 (2014).

68. McKinney, S.A., Joo, C. \& Ha, T. Analysis of single-molecule FRET trajectories using hidden Markov modeling. Biophys J 91, 1941-51 (2006).

69. Salsi, E., Farah, E. \& Ermolenko, D.N. EF-G Activation by Phosphate Analogs. J Mol Biol 428, 2248-2258 (2016).

70. Lu, Z.J., Gloor, J.W. \& Mathews, D.H. Improved RNA secondary structure prediction by maximizing expected pair accuracy. RNA 15, 1805-13 (2009).

71. Mathews, D.H., Burkard, M.E., Freier, S.M., Wyatt, J.R. \& Turner, D.H. Predicting oligonucleotide affinity to nucleic acid targets. RNA 5, 1458-69 (1999). 